\title{
Density profile characterization and modeling at Paranal and Armazones 2k sites
}

\author{
Julio C. Marín ${ }^{1,2, \star}$, Omar Cuevas $3,2, \star \star$, and Diana Pozo ${ }^{1,2, \star \star \star}$ \\ ${ }^{1}$ Departamento de Meteorología, Av. Gran Bretaña 644, Playa Ancha, Valparaíso, Chile \\ ${ }^{2}$ Centro de Estudios Atmosféricos y Astroestadística, Fac. Ciencias, Universidad de Valparaíso, Chile \\ ${ }^{3}$ Instituto de Física y Astronomía, Av. Gran Bretaña 1111, Playa Ancha, Valparaíso, Chile
}

\begin{abstract}
The Cherenkov Telescope Array (CTA) in the southern hemisphere will be installed at Armazones $2 \mathrm{k}$ site in northern Chile. Scarce atmospheric observations are available in the region, particularly radiosonde data. This study analyzes radiosondes launched at Paranal observatory, located at about $21 \mathrm{~km}$ from the CTA site, from 24 October and 4 November 2011, to understand the behavior of density in the atmosphere near the CTA site. High-resolution numerical simulations with the Weather Research and Forecasting (WRF) model are validated with Paranal radiosondes to quantify its ability to represent the atmospheric conditions in the region. In addition, the seasonal and diurnal evolution of atmospheric density at the CTA site were studied during 2011 using the high-resolution weather forecasts from the WRF model.
\end{abstract}

\section{Introduction}

A number of ground-based imaging atmospheric Cherenkov telescopes (IACTs) have been installed (H.E.S.S. [1]), VERITAS [2]) and MAGIC [3]) to observe gamma rays from galactic and extragalactic sources. The Cherenkov Telescope Array ((CTA) [4]) project is a large array of next-generation very high energy gamma ray telescopes that will improve the sensitivity of the gamma ray domain by installing two arrays in each hemisphere to cover the full sky. The one in the southern hemisphere will be located at Armazones 2k site in northern Chile (Fig. 1).

The atmosphere is used as a calorimeter in groundbased Cherenkov telescopes. Atmospheric density profiles affect the detection of air showers and the transmission of Cherenkov light through the atmosphere. For example, [5] found a $60 \%$ difference in Cherenkov light yield between tropical and polar atmospheric profiles, and $15-20 \%$ differences at a mid-latitude site due to seasonal variations in atmospheric profiles. Measurements from a radiosonde campaign will provide the information to reduce the uncertainties in the models used to simulate the Cherenkov light at the CTA site. In addition, radiosondes can be used to validate global or mesoscale models, which have been used to characterize sites hosting experiments to detect high energy cosmic rays ([6], [7]).

The arid northern Chile is a region with scarce atmospheric observations. This work aims to provide some information on the atmospheric characteristics at and near

\footnotetext{
$\star$ e-mail: julio.marin@meteo.uv.cl

$\star \star$ e-mail: omar.cuevas@uv.cl

$\star \star \star$ e-mail: diana.pozo@meteo.uv.cl
}

the southern CTA site. In order to do that: 1) we will analyze density profiles from radiosondes launched at a nearby site to CTA (Paranal Observatory), 2) validate high-resolution numerical weather simulations with Paranal radiosondes, and 3) study the diurnal and seasonal evolution of the atmospheric density at Armazones 2k site using high-resolution weather forecasts.

\section{Data and methodology}

\subsection{Radiosonde campaign at Paranal observatory}

The Paranal observatory is located at about $21 \mathrm{~km}$ from Armazones 2k site, in the North of Chile. Scarce meteorological observations make difficult to study the region. A radiosondes campaign was conducted from 24 October to 4 November 2011 at Paranal observatory, where 21 RS92SGP radiosondes were launched at 00 and 12 UTC. In this study, 19 radiosondes were used: 8 launched at 00 UTC and 11 launched at 12 UTC.

\subsection{WRF model configuration}

The WRF model is a mesoscale numerical weather prediction system that is used both for operational forecasting and atmospheric research. Version 3 of the model [8] was used in this study. The WRF model was run fully compressible, non-hydrostatic, with four nested domains (Fig. 1) and 69 vertical levels. This study evaluates the domain with higher horizontal resolution (d04 at $1 \mathrm{~km})$ centered on the Paranal observatory site. Global Forecast System (GFS) forecasts provided the initial and boundary conditions for WRF simulations every 3 hours. Simulations 


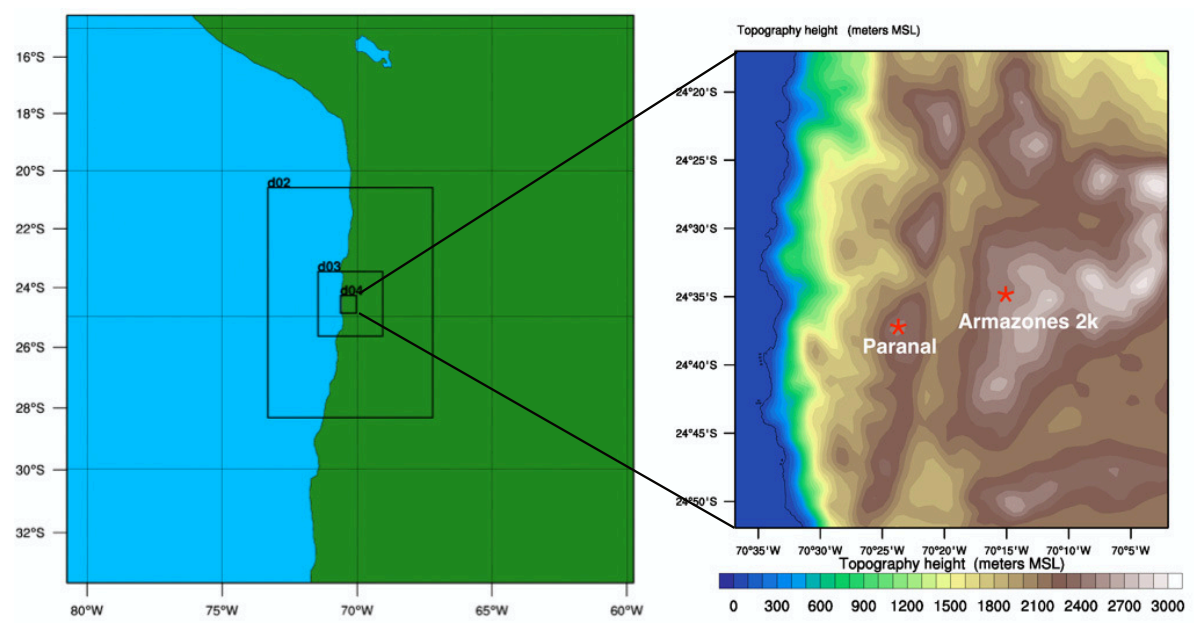

Figure 1. Left: The four nested domains used in WRF simulations in the study. Right panel: zoomed-view of the innermost domain (d4) showing the locations of Paranal and Armazones 2k sites.

were run every day for 24 hours, completing 315 days of 2011. Domain d04 at $1 \mathrm{~km}$ horizontal resolution was used in the analysis presented below.

\section{Results}

\subsection{Radiosondes analysis}

Figure 2a shows the average density profile and Fig. 2b the standard deviations from it. These are found to be two orders of magnitude smaller than the mean value at each level. When we analyze the relative variation of density standard deviation at each level compared to the averaged value, the fluctuations are typically less than $1 \%$ of the mean value, except between 15 and $20 \mathrm{~km}$ height (Fig. 2c).
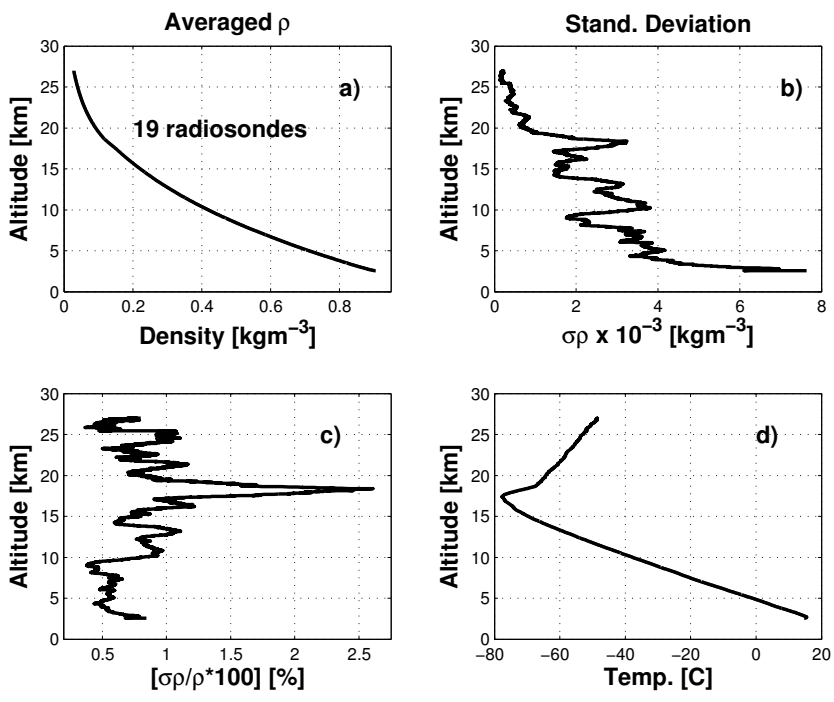

Figure 2. a) Average density profile, b) density standard deviation profile from the mean value, c) density standard deviation relative variation from the mean value and d) averaged temperature profile from radiosondes launched at Paranal.
A closer look at the layer 15-22 km shows that temperature profiles observed during the radiosonde campaign varied almost $20 \mathrm{~K}$ close to $18 \mathrm{~km}$ (Fig. 3). Therefore, the large relative density fluctuations observed in that layer are due to the large temperature variations that occurred near the tropopause level. The RS92-SGP radiosondes measure temperatures in the range $-90^{\circ} \mathrm{C}$ to $+60^{\circ} \mathrm{C}$ with a resolution of $0.1{ }^{\circ} \mathrm{C}$. The recorded temperatures near the tropopause are inside the technical specifications. However, the horizontal displacement of radiosondes at $18 \mathrm{~km}$ ranged between 45 and $100 \mathrm{~km}$, which could be one of the reasons of the large observed temperature variations.

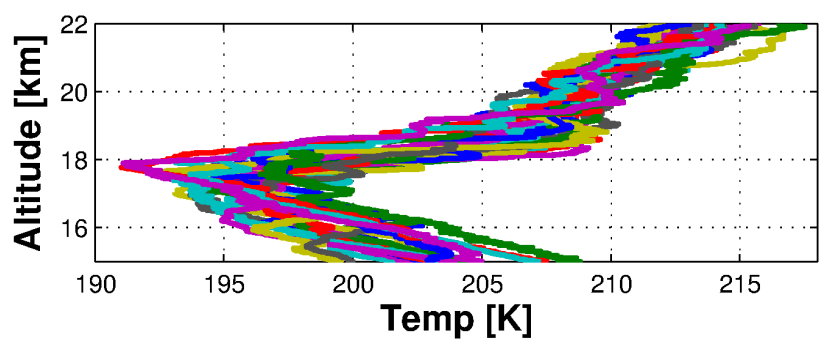

Figure 3. Zoomed view between 15 and $22 \mathrm{~km}$ height of temperature profiles measured by 19 radiosondes launched at Paranal during the period of study.

\subsection{Comparison between WRF and radiosondes}

Density profiles calculated from WRF simulations at the nearest grid-point to Paranal were compared to radiosondes. Just 12 radiosondes were used in the comparison since simulations were not available for some days of the study period. The mean density profile from simulations show close agreement with observations (Fig. 4a) from the surface to $20 \mathrm{~km}$ height.

The maximum density relative error between simulations and observations is plotted at each level from the surface to $20 \mathrm{~km}$ to analyze the maximum deviation from 

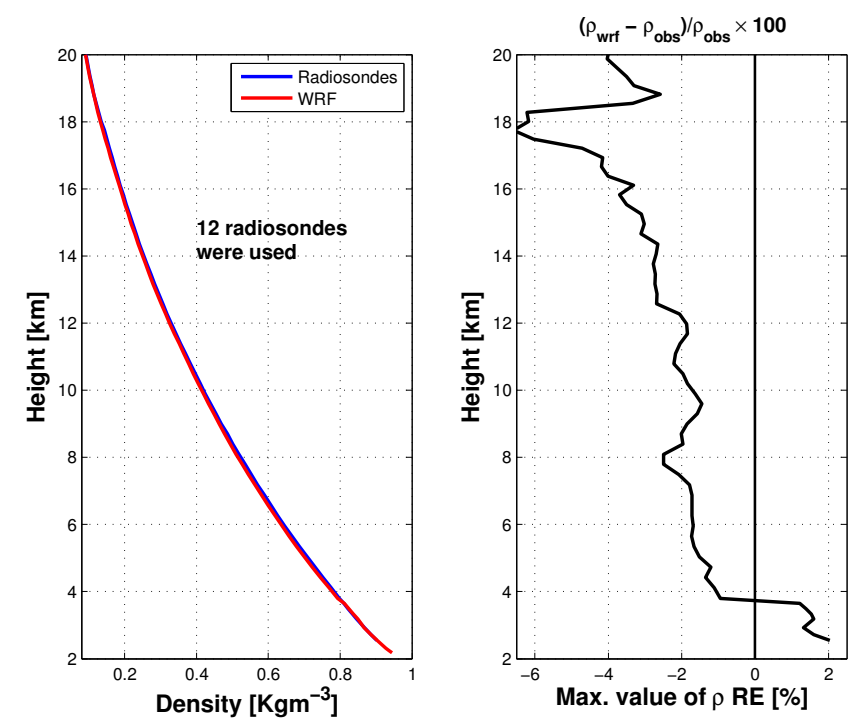

Figure 4. a) Averaged density profiles from WRF simulations and radiosondes and b) maximum density relative error at each level during the period of study.

observations that can be expected from WRF simulations (Fig. 4b). The model overestimates the atmospheric density below $4 \mathrm{~km}$ height and underestimates it above that altitude. Maximum absolute density relative errors less than $3 \%$ are shown in the troposphere. The relative error can increase close to $7 \%$ at the tropopause and lower stratosphere (15-19 km height), where the largest density relative variations seems to occur.

\subsection{Density seasonal and diurnal evolution}

Since the WRF model represents well the observed density profiles at Paranal site, WRF simulations were used to analyze the seasonal and diurnal evolution of density at the CTA site, where no radiosonde measurements are currently available.

The temporal evolution of simulated density in 2011 shows larger absolute variations at lower than at higher levels in the atmosphere (Fig. 5).

The relative density variation $(\rho-\rho($ year $)) / \rho($ year $) \times$ 100 was calculated from WRF simulations (Fig. 6). Its evolution during 2011 shows a clear seasonal trend at all levels in the atmosphere. Density decreases in austral spring and summer months and increases in fall and winter months below the tropopause level. On the other hand, a reverse variation is shown at the highest levels. Density increases in spring and summer months and decreases at fall and winter months. The largest density relative variations occurs near the tropopause and lower stratosphere. At those heights, large density relative variations can be seen in short periods of time, particularly during winter and spring months.

We grouped the simulated density profiles by season and calculated its relative variation over the year $((\rho$ (season $)-\rho($ year $)) / \rho($ year $) \times 100)$. The seasonal density profile varies less than $1 \%$ relative to the annual mean

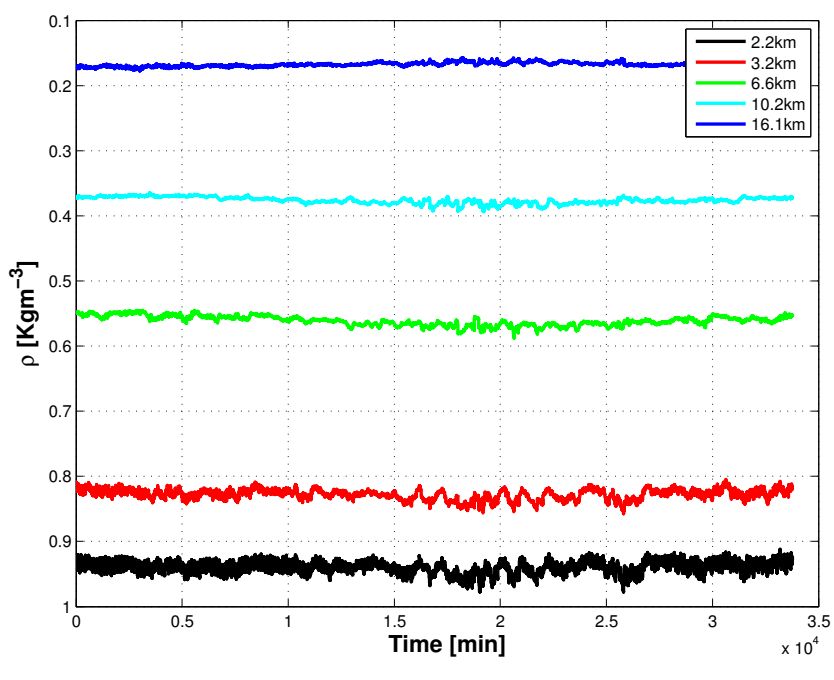

Figure 5. Temporal evolution of simulated atmospheric density from the nearest grid-point to the CTA site at different levels in the atmosphere (see legend for details).

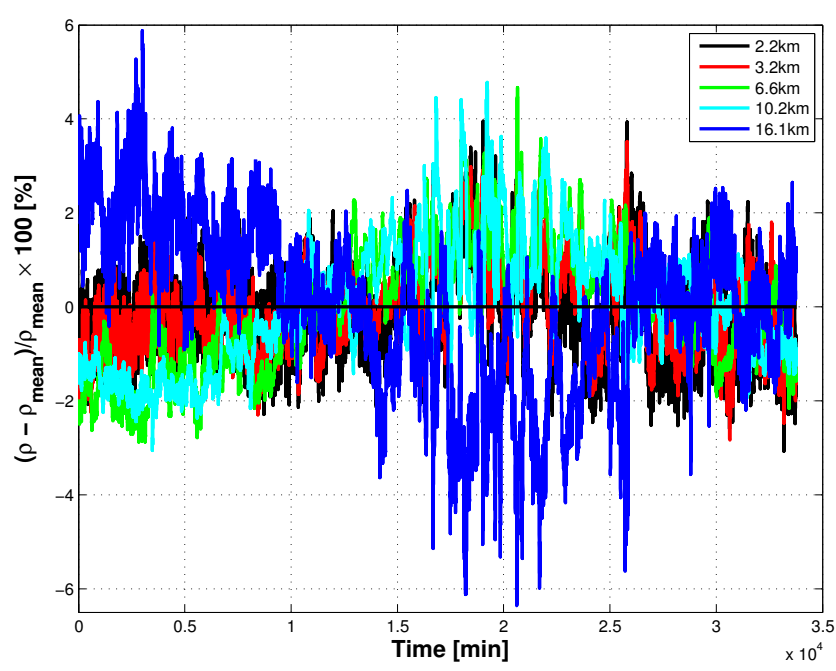

Figure 6. Temporal evolution of relative density variation $(\rho-$ $\rho$ (mean $)) / \rho$ (mean) from WRF simulations at the nearest gridpoint to the CTA site at different levels in the atmosphere (see legend for details).

within the troposphere but in the tropopause and lower stratosphere the seasonal density variation is larger than $3 \%$ of the annual mean (Fig. 7). The largest density relative variations occur in summer and winter months.

The diurnal evolution of density was studied by calculating the relative density variation at each hour compared with the annual mean. Fig. 8 shows that the largest density relative variations occur near the surface and the lower stratosphere during the day. The lowest density relative variations are shown during afternoon hours and the largest density relative variations are shown between 2-8 $\mathrm{h}$ local time. 


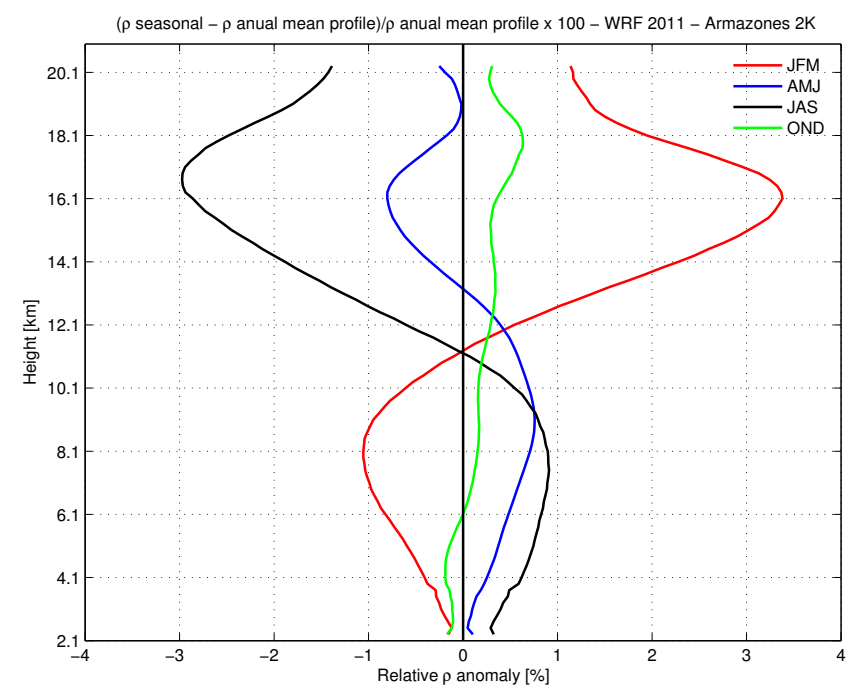

Figure 7. Relative seasonal density deviations from annual mean profiles from WRF simulations at the nearest grid-point to the CTA site (see legend for details).

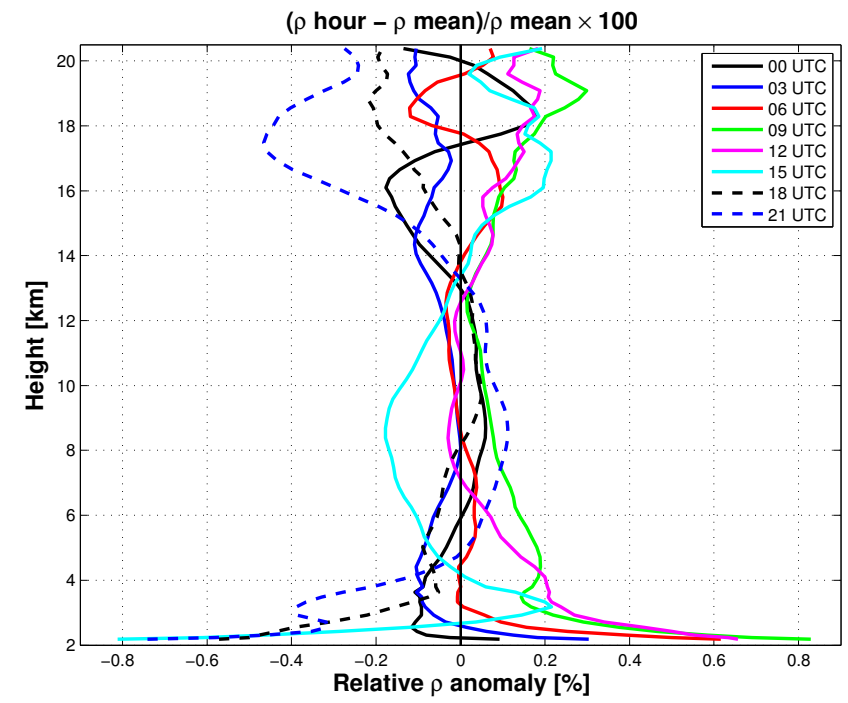

Figure 8. Three-hourly relative density deviations from annual mean profiles from WRF simulations at the nearest grid-point to the CTA site (see legend for details).

\section{Conclusions}

The Cherenkov Telescope Array (CTA) in the southern hemisphere will be installed at Armazones 2k site in northern Chile. Scarce atmospheric observations are available in the region, particularly radiosonde data. However, a small radiosonde campaign was conducted at Paranal observatory, located at about $21 \mathrm{~km}$ from the CTA site, from 24 October and 4 November 2011.

Radiosonde observations at Paranal showed that the largest density variability occurs near the tropopause, as a result of temperature changes. Figure $1 b$ of [9] also showed the largest variations in density profiles to occur between 10 and $20 \mathrm{~km}$ depending on location and season.

High-resolution numerical simulations with the Weather Research and Forecasting (WRF) model were performed almost every day in 2011 to provide weather forecasts for the region of study. Simulations were compared with radiosondes launched at Paranal observatory. Maximum relative errors between WRF and observations were less than $2 \%$ in the troposphere but slightly increase at the tropopause and lower stratosphere. Despite the good agreement between the model and observations, more observations are needed to have more confidence on the model results in order to use it in the region.

The simulated density is used to study its seasonal and diurnal variation at the CTA site. Absolute variations at Armazones $2 \mathrm{k}$ site are larger near the surface than at higher levels. In addition, density relative variations are larger in the lower stratosphere.

Density relative variations in the lower stratosphere increase in summer (up to 5\%) and during the day (up to $0.3 \%$ ) and decrease in winter (up to $-6 \%$ ) and during afternoon hours (up to $-0.45 \%$ ) at Armazones $2 \mathrm{k}$. The simulated density relative variations near the surface increase in winter (up to $4 \%$ ) and near dawn (up to $0.82 \%$ ) and decrease in summer (about $-3 \%$ ) and during afternoon hours (about -0.8\%) at Armazones 2k.

\section{Acknowledgements}

We acknowledge the European Southern Observatory (ESO) and Paranal staff for its support during the radiosonde campaign at Paranal site. Global Forecast System (GFS) model forecasts provided the initial and boundary conditions for the WRF model and were downloaded from the National Centers for Environmental Prediction (NCEP) servers. We acknowledge the Centro de Estudios Atmosféricos y Astroestadística (CEAAS) from Universidad de Valparaíso.

\section{References}

[1] https://www.mpi-hd.mpg.de/hfm/HESS/

[2] http://veritas.sao.arizona.edu/

[3] https://magic.mppmu.mpg.de/

[4] Actis, M., et al., Experimental Astronomy, 32, 193-316 (2011)

[5] Bernlöhr, K, Astroparticle Physics, 12, 255-268 (2000).

[6] Louedec, K., and M. Will, J. Phys.: Conf. Ser. 409, 1, (2013).

[7] Abreu, P., et al., Astroparticle Physics, 35, 9, 591-607 (2012)

[8] Skamarock WC, et al., NCAR/TN-468+STR NCAR TECHNICAL NOTE (2008)

[9] Daniel, M.K., Journal of Physics: Conference Series, 595 (2015) 